\section{TREATMENT OF AIR-RAID GASUALTIES}

BY

\section{S. JOSEPH HOFFMAN, M.B., F.R.G.S.Ed.}

It is not proposed to advance an excuse for adding to the literature that has accumulated on the treatment of air-raid casualties. The subject is one of importance, and some benefit may accrue from the consideration of a routine technique that was gradually evolved during a year's experience of numerous raids while R.S.O. at a hospital in the south of England and later consolidated elsewhere. The total number of casualties thus far treated exceeds 700 .

\section{Method of Reception of Casualties}

Early raids were fortunately on a small scale, casualties rarely exceeding fifty. Yet even these comparatively small numbers placed:a severe strain on the elaborate schemes that had been evolved for the reception of patients. In theory the concept of resuscitation wards, recovery wards, hysteria wards, " awaiting $x$-ray" wards, etc., was most attractive ; in actual practice it proved a complete failure. The same applied to the marking of patients with different colours to signify type and degree of injury, and urgency or otherwise of treatment. In the heat of the moment one never seemed able to remember what colour stood for what.

All previous schemes were therefore scrapped, and the existing facilities for dealing with " ordinary " accidents were merely amplified. Ali cases were admitted through the accident room in the usual manner. Here a rapid but comprehensive examination was made, and the findings were jotted down on a card, which was pinned to the patient's clothing. There was no attempt to decide whether operative intervention was urgently required or not, as this was deemed to lie within the province of the operating surgeon.

A careful search was made for the presence of a tourniquet, and if this was found it was removed forthwith. Usually little or no bleeding follows even when limbs have been blown off. This is because the elastic coat of the artery retracts and plugs the vessel, and the veins twist up on themselves. In the rare case in which haemorrhage was still brisk-as when a vessel had been half divided and so could not retract-an attempt was made to control it with artery forceps. If this succeeded the tourniquet was left off; otherwise it was reapplied. Finally a firm bandage over wool was applied, and this almost always controlled all bleeding. A.T.S. 2,000 units was administered as a routine, together with morphine, and the patient was then transferred to the ward. The time-honoured dosage of morphine grain $1 / 4$ is almost without effect; a minimum of $1 / 2$ grain and often 1 grain is required. In particularly restless and psychically shocked patients this is best given intravenously. The injection must be made very slowly, and $1 / 2$ grain is the maximum that can be safely given in this way. The effect is almost instantaneous.

\section{Pre-operative Treatment}

Every casualty is suffering from shock and requires adequate anti-shock treatment before operation. This fact now seems axiomatic. But in the first flush of enthusiasm one tended to overlook it and to rush cases into the theatre prematurely. This was particularly so with wounds of the thorax or abdomen. It resulted in successful operations but, unfortunately, in dead patients. In fact, after the first raid our mortality rate was over $40 \%$, in spite of every operation having been completed within six hours of the raid.
On reaching the ward the patient is transferred to bed between warmed blankets, and anti-shock treatment is started. No attempt is made to undress the patient, the clothing being merely loosened where tight. If it is necessary to expose any area the overlying clothing is cut away. Every effort is made to disturb the patient as little as possible. As a preliminary measure the blood pressure is taken. It is invaluable both as a prognostic measure and as an index of response to treatment and fitness for operation. A systolic pressure below 80 almost always means that anti-shock treatment will fail and that death will ensue.

\section{Measures against Shock}

The anti-shock measures are best described in the following categories :

1. Rest (both Mental and Physical).-This is attained with the morphine. If the dosage so far given is ineffective the drug is repeated forthwith, preferably intravenously in order to get an immediate effect. At the same time temporary splints are adjusted so as to ensure immobilization of injured limbs.

2. Heat.-This is best attained with a radiant-heat cradle. Cradles were made in bulk by the hospital electrician at a cost of some 30s. each.

3. Fluid Replacement by Venoclysis. - The solution of choice is plasma. It is put up in vacoliters, and the assembly of the apparatus and introduction of the needle into a vein take but a few seconds. In a moderately severe case at least four pints is required. This is to be regarded as $a$ basic minimum and may be insufficient. It is given as a fast drip at room temperature. It must be stressed that it is useless to pour in plasma at one end if it is leaking out almost as fast at the seat of injury. Literally pints can be lost through a lacerated wound of moderate severity within a few hours. Therefore a vigilant eye is kept on dressings, and if these become moist they are immediately taken off and the wound inspected. If necessary a tourniquet is tightly applied just above the site of injury, and this effectively prevents any further plasma leak. Naturally the tourniquet is loosened at twenty-minute intervals.

4. Oxygen Therapy.-This is an important adjuvant in the treatment, and quite dramatic improvement may follow the correction of tissue anoxaemia. The oxygen is best given through the B.L.B. mask. This latter is practically foolproof and, once adjusted, requires a minimum of attention. The technique of application, too, is simple. But one tip to the tyro: Use either a flowmeter or else a full oxygen cylinder.

\section{Operative Treatment}

This is undertaken as soon as the maximum response is thought to have been obtained from the anti-shock therapy. It is essentially a question of surgical judgment; but in the borderline case the blood pressure gives invaluable aid. To operate while the systolic pressure is still under 100 is to court disaster. It must be borne in mind, however, that with fractures of the long bones complete recovery from the shock is not obtained until the displacement has been rectified. If thought necessary the venoclysis is continued throughout the operation.

The choice of anaesthetic is important. My preference is for sodium pentothal. I would remind those who use it, however, that the specific antidote is picrotoxin, and wou!d suggest that ampoules of the latter kept on the anaesthetic table may prove life-saving.

\section{rechnique}

This naturally varies with the site and extent of the injury. However, in all cases certain principles are observed :

(a) No more is done than is essential to save life. After all, that is the prime function of the traumatic surgeon. Therefore one errs on the side of conservatism, deferring all procedures not essential to this end until a later date. (b) Primary suturs of anything except clean incised wounds is 
never done. (c) Amputation is rarely performed as a primary procedure unles: the blood supply is irretrievably lost.

The usual type of injury commonly involves the limbs, and it is proposed to describe the method used in dealing with such a lesion:

1. The wound is exposed, and the surrounding healthy skin (from the joint above to the joint below) is scrubbed with soap-and-water and then painted with iodine.

2. The wound itself is scrubbed with a brush and dilute lysol water-2 drachms to the pint. This stains all dead tissue a dirty greyish white, and makes its subsequent recognition easy.

3. The wound is flooded with hydrogen peroxide, and this in turn is washed out with spirit or ether.

4. All dead tissue is now excised, beginning with the skin and working inward. A minimum of skin edge is removed. An effort is made to remove all foreign bodies; and the generally expressed view, that most metallic foreign bodies can be safely left, is not agreed with. They are a potent source of chronic wound sepsis, and may be one of the predisposing factors in the development of gas gangrene. Efficiency in locating and removing pieces of shrapnel comes only with experience. The wound is excised down to the deep fascia, and the point of penetration here is easily recognized. This is excised, and a finger is now gently insinuated into the track, and the latter is followed down as far as the finger will go. If the shrapnel is still not reached, a second incision is made on to the finger-tip; the finger is withdrawn from the track, reinserted through the new opening, and the track again followed up. This procedure is repeated, if necessary, until eventually the shrapnel is felt, when it is removed with a pair of forceps. It is necessary to stress the need for the utmost gentleness. "False passages" in the muscles are humiliatingly easy to make, and once one gets outside the original track it is well-nigh impossible to get back. By this means it is possible to trace a missile all the way up from the knee to the thigh with the minimum of tissue disturbance. In point of fact $I$ have vivid memories of watching my ex-chief (who was an Army surgeon in the war of 1914-18) doing so, and producing a piece of metal the size of a golf ball from beneath the upper end of the adductor magnus-the entrance wound being in the region of the knee. No mention has been made of radiological localization. It was not found practicable to radiograph more than some $5 \%$ of cases. The time factor, and also sheer weight of numbers, precluded the use of this aid except where considered essential.

5. Toilet having been completed, the wound has a guttered appearance, being almost as wide in its depths as on the surface. Large bleeding-points are tied off, but all others are left, the aim being to reduce to an absolute minimum the foreign material (in this case catgut) in the wound.

6. Finally, the wound is well smeared with "zisp" and then packed with gauze impregnated with the same material. The bone ends, if severed, are manipulated into position, and the $\operatorname{limb}^{\circ}$ is immobilized in plaster-of-Paris. Primary suture of the wound is virtually never performed.

"Zisp " calls for some explanation. Its formula is :

$$
\begin{aligned}
& \text { Zinc peroxide } \\
& \text { lodoform } \\
& \text { Sulphanilamide powder } \\
& \text { l'araffin, q.s. to make a thin paste. }
\end{aligned}
$$

The mechanical action is similar to that of the petroleum-jelly gauze in the Winnett Orr treatment, but there would appear to be several decided advantages. The emulsion is a deodorant. Thus there is none of the fetid odour which emanates from the patient subject to the Winnett Orr method, and it is possible to retain the plaster for as long as desired. The zinc peroxide liberates oxygen, and so retards the growth of anaerobic organisms. The inclusion of the sulphanilamide needs no comment. In spite of foreign views to the contrary, most surgeons agree that it plays a large part in keeping wounds aseptic.

\section{Post-operative Treatment}

This is virtually the same as originally recommended by Winnett Orr, except that it is not necessary to change the plaster because of the smell. One is guided by the general condition, by the pulse and the temperature, and by the local condition as evidenced by pain. Usually the temperature and pulse are normal, and the patient is comfortable within two days. Persistent pain and a rapid pulse are each an indication par excellence for removal of the plaster and inspection of the wound.

A final word about the late management of the wound. Frequently it does very well for a time, and then inexplicably hangs fire. It is at this stage that small doses of insulin may work miracles. Five units night and morning suffices.

\section{Question of Gas Gangrene}

I have had the misfortune to see eleven cases of gas gangrene since the war began. Clinically these can best be divided into two groups, although in all cases the causative organisms were discovered.

\section{Mild Cases}

These usually occur in wounds which have been subjected to inadequate toilet and to primary suture. The patient is moderately toxic, with a temperature around $102^{\circ}$ and a pulse rate around 120 . The suture line is obviously under tension, and the wound is resonant to percussion. On removal of the sutures a thin dirty brown fluid, teeming with anaerobic Clostridia, seeps out. In this type a $100 \%$ cure can be expected from a combination of relief of tension, deep therapy, and chemotherapy. Ablation is never required.

\section{Severe Cases}

Here the clinical picture is a different one. The patient is acutely ill and highly toxic. The temperature is not high, but the pulse is rapid and thready. The mental state is a charac. teristic one, the patient being hyperalert, and probably asserting that he had never felt better. Locally the limb has a tense drum-like appearance and characteristic odour. The skin has a mottled marbled appearance with typical venous marking. The arteries are not palpable. This is a true gangrene and the blood supply is occluded, though whether this latter be cause or effect is problematical. It spreads almost as one watches it, and surgery should be considered both urgent and imperative. Local intervention is meddle. some, and in immediate ablation lies the only hope.

At operation the limb is removed by a transverse incision, but the bone is divided as high above the skin level as possible. No attempt is made to get above the upper limit of infection, if necessary one being quite satisfied to go through the typical greyish mushy muscle. Haemorrhage is scanty. and only the main vessels are tied off. The wound is then packed with acriflavine in paraffin gauze, and the pack is held in position by three or four skin sutures

Post-operative treatment consists in a combination of intensive chemotherapy with sulphapyridine, deep therapy, venoclysis of glucose in saline, and anti-gas-gangrene serum. Three of the four cases of this type that I was associated with recovered on this regime. Four days later the skin sutures are divided and the pack removed. The wound now presents an amazing appearance. It literally looks as if it has just been made. This is because the acriflavine retards the formation of granulation tissue. Under local anaesthesia secondary suture is performed just as though it were a fresh wound, and healing virtually occurs by first intention. This method of performing the amputation is as fast as the true guillotine operation and has none of its disadvantages.

My thanks are due to Mr. H. John Nightingale, senior surgeon to the Royal South Hants Hospital, who is responsible both for my interest in traumatic surgery and for teaching me its principles and practice.

The annual general meeting of the Royal Medical Benevolent Fund was held at the house of the Royal Society of Medicine on May 14, when Sir Thomas Barlow, Bt., was re-elected President, Dr. Lewis G. Glover Honorary Treasurer, and $\mathrm{Mr}$. R. M. Handfield-Jones Honorary Secretary. The accounts for the year ended December 31, 1940, were presented and adopted for circulation to subscribers. 